\title{
Using Two Sensors for Damage Detection of Bridge Based on Transfer Entropy of Vehicle-bridge Information
}

\author{
Julei Zhu' ${ }^{1}$, Wen Cai $^{2}$ \\ 1. Jinan University, Guangzhou, 510632, China \\ 2. The Key Laboratory of Disaster Forecast and Control in Engineering, Guangzhou, 510632, China
}

\begin{abstract}
An innovative damage identification method using only two sensors for damage detection of bridge based on transfer entropy of vehicle- bridge information is proposed in this article. In this method, a test vehicle passing through the bridge surface, and structural vibration acceleration responses at two measuring points are measured from two acceleration sensors, which one set on the bridge, and the other one set on the test vehicle. A fixed moving window is defined as a definitive length calculated by the sampling frequency and the fundamental frequency of the measured acceleration responses. The windowed pair time series extracted from the two measured acceleration responses that obtained from the bridge and the moving vehicle respectively are used to calculate the transfer entropy, which is used to define the new damage index. A simply supported beam bridge subjected to a test vehicle is simulated to verify the effectiveness and feasibility of this method. Numerical simulation results show that this method can accurately detect the damages under both single damage scenarios and multiple damage scenarios using acceleration responses obtained from bridge and moving vehicle. It indicates that the method proposed in this article has a promising application prospect in the field of bridge damage detection.
\end{abstract}

Key words: structural damage detection; transfer entropy; two sensors; moving vehicle

\section{Introduction}

Due to corrosion and material aging, the performance of bridge structure is reduced or even damaged, which seriously affects the normal operation of traffic and the safety of people's life and property. Therefore, bridge condition monitoring and damage identification of bridge structures are particularly important and have become a hot issue of current research.

At present, structural damage detection founded on dynamic response is the mainstream method and is suitable for large structures. However, traditional damage detection methods, such as the method of modal identification, require numerous sensors setting on the bridge, which is labor-intensive and uneconomical. Therefore, it is urgent to develop structural damage detection and health monitoring methods based on limited sensor information to reduce costs and meet the financial and monitoring needs of managers.

Methods based on limited sensors are rarely studied at present. Few sensors acquire less data, and modal analysis 
generally requires more sensors to identify the complete modal. Therefore, the method based on limited sensors can not start from a modal point of view, but extract effective information from the response signal of the structure, and discover information related to structural damage, so as to realize structural damage identification. In the last few years, researchers have attempted to detect structural damage with limited sensors, or even a single sensor. The method of using the response measured from single sensor to detect damage of the structural was proposed by Zhu and Law. They use only one sensor to measure the mid-span displacement signal of the beam and use Continuous Wavelet Transform (CWT) for estimating the damage degree of a simply supported beam under moving loads.

Nie et al. put forward a novel damage identification approach, which extracts the response data from a single sensor to identify damage by reconstructing phase space. The numerical simulation and experimental structure infer that this proposed approach can locate damage. Li proposed a damage detection method under the moving vehicle founded on a dynamic response reconstruction manner. These methods mentioned above acquiring the response data extracted from bridge through the sensors set on the bridge, and analyzing the acquired information to achieve the purpose of bridge damage identification. We collectively call these methods direct measurement methods.

Yang et al. (2004) presented the conception of indirect measurement method for the first time in the world. Instead of installing sensors directly on the bridge for obtaining the responses of the bridge, the proposed method installing sensors on the moving test vehicle passing through the beam for acquiring the vibration information of the bridge. The vehicle acts as both an excitation input to the bridge and also a data acquisition system to record vibration response information.Inspired by the bridge indirect measurement method proposed by Yang. In 2006, J.Q.Bu attempted to use indirect measurement method for damage identification of bridge structures. He constructed the damage index by changing the rigidity of the bridge unit and proposed a sensitivity analysis approach to detect the bridge damage using the acceleration signal of the moving vehicle. In 2010, Nguyen and Tran studied the displacement signal of the moving vehicle and the damage judgment of the bridge structure using wavelet analysis technology. It was found that the greater the cracking depth, the easier the damage identification was. Meanwhile, the damage detection results of the test vehicles at low speeds have better accuracy. These methods mentioned above have some disadvantages in engineering application, such as inadequate accuracy and robustness to noise.

Shannon proposed the concept of information entropy in 1948, which was used to measure the degree of uncertainty in variables. Schreiber put forward the concept of transfer entropy based on Shannon Entropy and time delay interactive information in 2000. It is an important aspect of information entropy theory. Transfer entropy is essentially constructed from two or more information entropies, so it has all the characteristics of information entropy. Transfer entropy, as a component of information entropy theory, has been widely used in various fields, but its application in engineering is still in its initial stage. In 2006, Nichols applied transfer entropy theory to damage identification of simple mechanical structures. Thomas Dimpfl validated that the transfer entropy theory is also applicable to nonlinear and linear systems, which laid a foundation for the application of the transfer entropy theory in engineering.

According to the indirect measurement theory, the dynamic characteristics of the bridge can be taken out from the measured responses of the test vehicle. In this method, a test vehicle passing through the bridge surface, and structural vibration acceleration responses at measuring points are measured from two acceleration sensors, which one sett on the bridge, and the other one set on the test vehicle. By making full use of the characteristic of transfer entropy, the correlation degree between different information flows can be estimated and the transfer relationship between information flows can be quantified. The transfer entropy value of the response information acquiring from the two sensors in the vehicle-bridge system can be calculated to achieve the purpose of damage detection. The feasibility and accuracy of this method are 
verified by numerical simulation of a simply supported beam bridge model.

\section{Method}

2.1 Dynamic response analysis of a simply supported beam bridge subjected to a test vehicle

Yang developed the vehicle-bridge interaction unit, called VBI (Bridge-Vehicle Interaction) unit, to study the coupling vibration of high-speed train and bridge. The two systems are connected by the vehicle-bridge interaction force and the slow calculation speed is solved by iteration. To spotlight the main dynamic characteristics of the coupled vehicle-bridge system, a simplified mathematical model will be accepted, as show in Figure 1. The test vehicle with the speed of $v$ passes through a beam bridge. The test vehicle is simulated by a moving spring-mass model and the bridge is simulated by an Euler-Bernoulli beam. As show in Figure 1, the model parameters are as follows: $m_{v}$ denotes the suspension mass of the test vehicle, ${ }_{v}$ is the spring stiffness of the vehicle, and $v$ is the velocity of the test vehicle. $L$ denotes the length of the simply supported beam bridge. $\bar{m}$ is the mass per unit length of the beam bridge. $E$ and $I$ denote the elastic modulus and moment of inertia of the beam bridge, respectively. Without considering the damping of the beam bridge and the test vehicle, the vibration equations of vehicle and bridge can be expressed as follows:

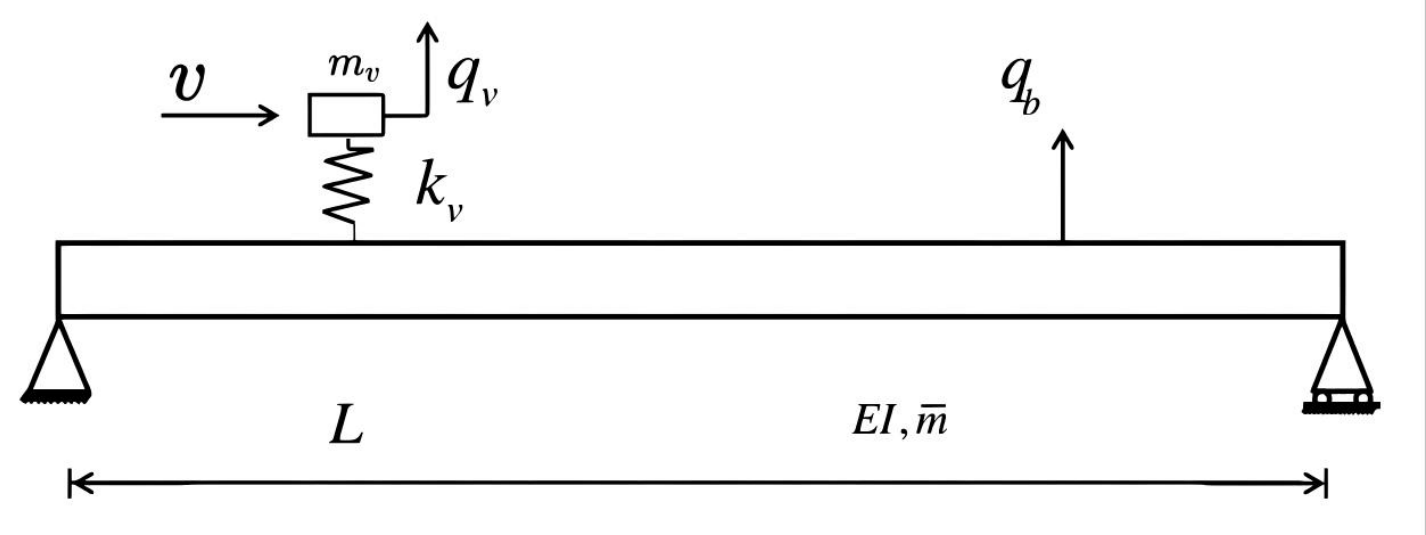

Figure 1. Model of simple-beam and passing vehicle based on indirect measurement method

$$
\begin{gathered}
\bar{m} \ddot{u}(x, t)+\operatorname{EIu}^{\prime \prime \prime}(x, t)=f_{c}(t) \delta(x-v t) \\
\mathrm{m}_{v} \ddot{q}_{v}(t)+k_{v}\left(q_{v}(t)-\left.u(x, t)\right|_{x=v t}\right)=0
\end{gathered}
$$

Where $u(x, t)$ denotes the vertical displacement of the beam bridge, $q_{v}(t)$ is the vertical displacement calculated from the equilibrium position when the vehicle is stationary, $\ddot{u}(x, t)$ is the twice differentiation of displacement against time, $u^{\prime \prime \prime}(x, t)$ is the fourth differentiation of displacement against the position $x$ of the test vehicle and $\delta$ is the delta function.

$f_{c}(t)$ in equation.(1) is the interaction force between the vehicle and the bridge, generated by the vehicle's self-weight and the relative displacement between the vehicle and the bridge, expressed as

$$
f_{c}(t)=-m_{v} g+k_{v}\left(q_{v}(t)-\left.u(x, t)\right|_{x=v t}\right)
$$

For the simple beam, the vertical displacement $u(x, t)$ can be expressed in terms of the modal shapes $\sin (n \pi x / L)$ as 


$$
u(x, t)=\sum_{n=1}^{\infty} \sin \frac{n \pi x}{L} q_{b, n}(t)
$$

Where $q_{b, n}(t)$ is the $n$th modal coordinate of the beam.

The solution of the displacement response of the moving test vehicle can be acquired as fallows (Lin et al 2004, Chang et al 2009):

$$
\begin{aligned}
q_{v}(t)=\sum_{n=1}^{\infty}\{ & A_{1, n} \cos \left(\frac{(n-1) \pi v}{L}\right) t+A_{2, n} \cos \left(\frac{(n+1) \pi v}{L}\right) t+A_{3, n} \cos \left(\omega_{v} t\right) \\
& \left.+A_{4, n} \cos \left(\omega_{b, n}-\frac{n \pi v}{L}\right) t+A_{5, n} \cos \left(\omega_{b, n}+\frac{n \pi v}{L}\right) t\right\}
\end{aligned}
$$

Where the vehicle frequency $\omega_{v}$, bridge frequency $\omega_{b, n}=\frac{n^{2} \pi^{2}}{L^{2}} \sqrt{\frac{E I}{\bar{m}}}$,

and $A_{1, n} 、 A_{2, n} 、 A_{3, n}, A_{4, n} 、 A_{5, n}$ are the amplitudes corresponding to each frequency.

From the displacement response of the test vehicle, we can see that the displacement response consists of the vibration response corresponding to the four frequency components $n \pi v / L, \omega_{v}, \omega_{b, n}+n \pi v / L, \omega_{b, n}-n \pi v / L$. This means that the response of the test vehicle does contain the response information of the bridge vibration, so it is feasible to obtain the vibration characteristics of the bridge by measuring and testing the response of the vehicle indirectly.

Differentiating equation (5) twice for time $t$, the acceleration response of the test vehicle can be obtained, and can be expressed as:

$$
\ddot{q}_{v}(t)=\sum_{n=1}^{n}\left\{\begin{array}{l}
\tilde{A}_{1, n} \cos \left(\frac{(n-1) \pi v}{L}\right) t+\tilde{A}_{2, n} \cos \left(\frac{(n+1) \pi v}{L}\right) t+\tilde{A}_{3, n} \cos \omega_{v} t \\
+\tilde{A}_{4, n} \cos \left(\omega_{b, n}-\frac{n \pi v}{L}\right) t+\tilde{A}_{5, n} \cos \left(\omega_{b, n}+\frac{n \pi v}{L}\right) t
\end{array}\right\}
$$

where $\tilde{A}_{1, n} 、 \widetilde{A}_{2, n} 、 \widetilde{A}_{3, n}, \widetilde{A}_{4, n} 、 \widetilde{A}_{5, n}$ are the amplitudes corresponding to each frequency.

As observed, the vehicle response is dominated by five frequencies, i.e., the left-shifted driving frequency $(n-1) \pi v / L$, right-shifted driving frequency $(n+1) \pi v / L$, vehicle frequency $\omega_{v}$, left-shifted bridge frequency $\omega_{b, n}-n \pi v / L$, and right-shifted bridge frequency $\omega_{b, n}+n \pi v / L$. This means that the response of the test vehicle does contain the response information of the bridge vibration, so it is feasible to obtain the vibration characteristics of the bridge by indirect measurement by testing the response signal of the vehicle.

\subsection{Transfer entropy theory}

The concept of information entropy was put forward by Shannon in 1948. The main measure of uncertainty in information is defined as

$$
Q=-\sum_{i=1}^{N_{m}} p_{i} \ln p_{i}
$$

Where $p_{i}$ denotes the probability of the $i$ th signal in the source, $\ln p_{i}$ is the amount of information that the 


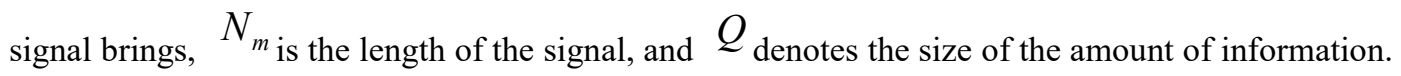

Transfer entropy is developed by Schreiber according to the concept of information entropy. At present, transfer entropy has become an important aspect of information entropy theory. Transfer entropy is composed of information entropy, its essence is still information entropy, so it has all the characteristics of information entropy. Transmission entropy refers to the calculation of information entropy between two signals. Therefore, singularity can be found by determining the amount of missing information between signals.

Firstly, two stationary Markov processes are given, which are represented by $x$ and $y$ respectively, and $M_{y \rightarrow x}$ denotes the transfer entropy of process $y$ to process $x$. In addition, in this hypothesis process, the historical information about $x$ is completely known. Assume that the probability that process $y$ affects process $x$ is $p$, the expression of transfer entropy of coupling relationship between two processes can be expressed as:

$$
\begin{gathered}
M_{y \rightarrow x}\left(x(1) \mid x^{(k)}, y^{(l)}\right)= \\
\left.\iiint p\left(x(1), x^{(k)}, y^{(l)}(\tau)\right) \log _{2} \frac{p\left(x(1) \mid x^{(k)}, y^{(l)}(\tau)\right)}{p\left(x(1) \mid x^{(k)}\right)}\right) \times d x(1) d x^{(k)} d y^{(l)}
\end{gathered}
$$

Where, $k 、 l$ present the order of two Markov processes $x$ and $y$, respectively, and $\tau$ is the delay time.

Nichols and Overbey have assumed that both process $x$ and process $y$ are first-order Markov processes, that is, $k=l=1$. This assumption can effectively avoid the complex calculation of high-dimensional probability density functions required in the calculation of formulas. Using the first order process will not affect the validity of studying the degree of coupling between the measurement processes of transfer entropy, which can greatly reduce the amount of calculation and bring great convenience to the calculation. Delay time $\tau$ in equation(8), which can be interpreted as the amount of information contained by process $y$ in relation to process $x$ at different time scales, i.e. the effect of process $y$ on process $x$ at different time scales. The meaning of transfer entropy is to quantify how process $y$ affects process $x$. It needs to be assumed that at some future time, the state of process $x$ is entirely dependent on its own history. If the state is determined, then the value of transfer entropy is 0 .

In practice, the dynamic response of most engineering building structures can be viewed as a linear dynamic process, and for this process, the equation for transferring entropy can be simplified. Firstly, two dynamic processes with linear and Gaussian distribution are listed, which are recorded as processes $x$ and $y$. The transfer entropy equation (9) can be conveniently expressed as the determinant product of variance and covariance matrix according to their linear and Gaussian characteristics:

$$
M_{y \rightarrow x}(x(1) \mid x, y(\tau))=\frac{1}{2} \log _{2} \frac{\left|c_{x(1) \mid x, y(\tau)}\right|\left|c_{x}\right|}{\left|c_{x, y(\tau)}\right|\left|c_{x(1), x}\right|}
$$

Where $\|$ in the logarithmic column is used to solve the matrix determinant, the other terms in the equation are solved as follows:

$$
\begin{gathered}
C_{x(1) \mid x, y(\tau)}=\left[\begin{array}{ccc}
E[x(n+1) x(n+1)] & E[x(n) x(n+1)] & E[y(n+\tau) x(n+1)] \\
E[x(n) x(n+1)] & E[x(n) x(n)] & E[x(n) y(n+\tau)] \\
E[y(n+\tau) x(n+1)] & E[y(n+\tau) x(n)] & E[y(n+\tau) y(n+\tau)]
\end{array}\right] \\
C_{x}=E[x(n) x(n)]=o_{x}^{2}
\end{gathered}
$$




$$
\begin{aligned}
C_{x, y(\tau)} & =\left[\begin{array}{cc}
E[x(n) x(n)] & E[x(n) y(n+\tau)] \\
E[y(n+\tau) x(n)] & E[y(n+\tau) y(n+\tau)]
\end{array}\right] \\
C_{x(1), x} & =\left[\begin{array}{cc}
E[x(n+1) x(n+1)] & E[x(n) x(n+1)] \\
E[x(n+1) x(n)] & E[x(n) x(n)]
\end{array}\right]
\end{aligned}
$$

Where $E$ denotes the expectation, $O$ is the standard deviation.

\subsection{Damage detection method of vehicle-bridge information transfer entropy}

The approach proposed in this article uses only two sensors that one set on the test vehicle passing through the bridge surface and the other set at any position of the beam bridge, as shown in Figure 2. The responses of the test vehicle and the beam bridge is measured under the action of the test vehicle simultaneously. Depending on whether the transfer entropy value between the acceleration response measured by the two sensors changes abruptly before and after the damage, the damage of the bridge can be judged and located accordingly.From the above deduction analysis, it can be seen that when the structure changes, the amount of information between the two measuring points will also change. Nevertheless, if the whole time series of these two responses is used for the transfer entropy calculation, only one value of transfer entropy is acquired, which can probably fail to reflect the local damage information hidden in the time series. Consequently, a moving window is defined to extract a certain length of time series response fragment from these two responses for transfer entropy analysis.

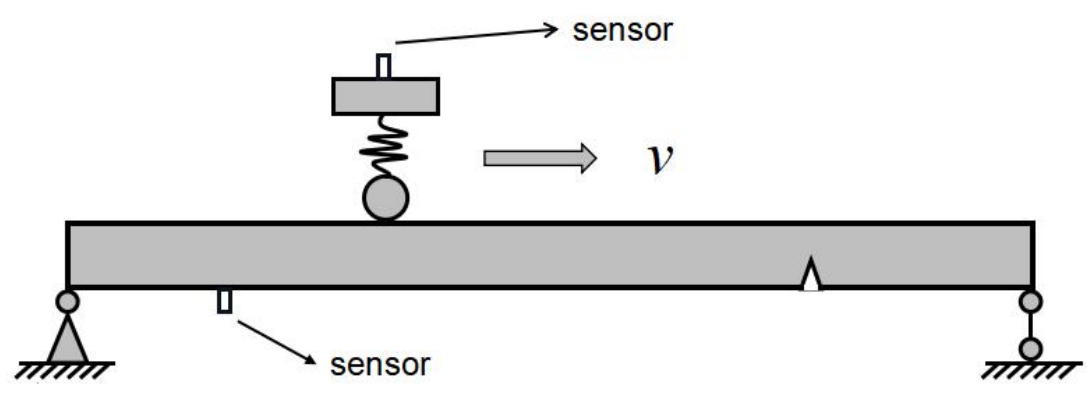

Figure 2. Schematic diagram of the simply supported beam bridge model and the test vehicle model

The window length is related to the fundamental frequency of the measured responses. A window with a moving length of $l$ is defined to separate the total length of the response data, facilitating the comparison of responses of two groups of data at different locations. The length $l$ can be expressed as:

$$
l=2 \frac{f_{s}}{f_{1}}
$$

$f_{s}$ denotes the sampling frequency of the response data and $f_{1}$ denotes the fundamental frequency of the response data.

Using the defined moving time window for the acceleration response of two measurement points from the moment $t=0$ for synchronous signal interception, as shown in Figure 3, the two segments within the window are calculated to obtain the transfer entropy value between the two segments within the window, and the time series of transfer entropy $M$ is obtained by sequentially moving the window with the length $N-l$. 


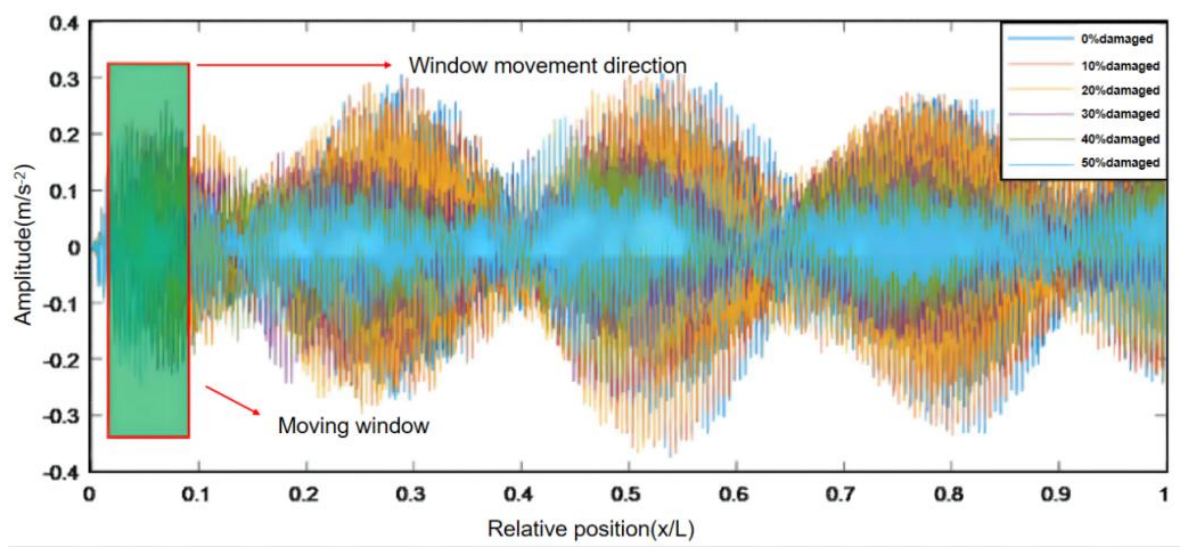

Figure 3. Moving window schematic

Calculating the transfer entropy of the two measurement points within the time series window according to the formula for the transfer entropy obtained above, the damage index $V$ can be expressed by the following equation (15):

$$
V_{i}=\frac{\sqrt{\sum|| M_{x_{i} y_{i}}^{d}|-| M_{x_{i} y_{i}}^{u} \mid}}{\sum\left|M_{x_{i} y_{i}}^{u}\right|}, i=(1 \cdots N-l)
$$

Where $M_{x_{i} y_{i}}^{d}$ denotes the data of transfer entropy between two measurement points under the damaged state within the window length. ${ }^{M_{x_{i} y_{i}}^{u}}$ is the data of transfer entropy between two measurement point under the undamaged state within the window length. Since the number of windows is $N-l$, the number of $V$ is also $N-l$.

Locating the damage by determining the peak of the damage index $V$ value curve. The flow chart of damage location method based on transfer entropy is shown in Figure 4: 
Step1: Setting one sensor at any position on the bridge and one on the vehicle

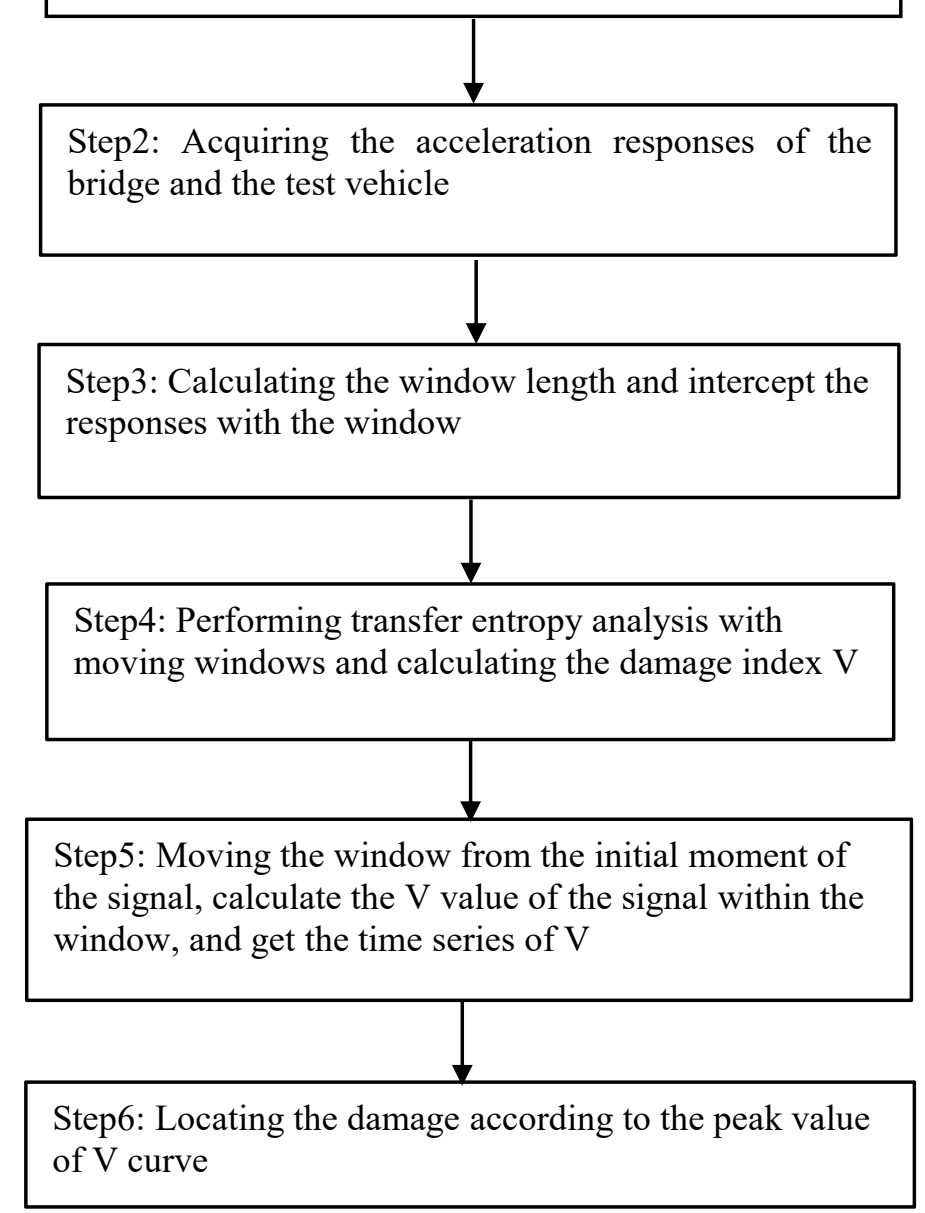

Figure 4. Calculation flow chart of the damage index V

\section{Numerical Simulation}

In order to verify the feasibility of this proposed method, a simply supported beam is selected as the calculation model in this article. The moving load is a single-degree-of-freedom vehicle model as shown in the Figure 5. In this paper, MATLAB software is used to analyze the vehicle-bridge coupling vibration model and verify it by programming numerical simulation. As shown in Figure 5, installing one sensor on the test vehicle, and the other marked as sensors 1 to 10 installed at the same distance from the left to right on the simply supported beam, and a small red triangle between sensors is marked as the location of damage as shown in the Figure 5. In order to verify the influence of using different sensor pairs on the damage identification results, ten sensors installed on the bridge, actually, only one sensor of the ten sensors marked as sensors 1 to 10 on the simple beam are chosen, and together with the sensor 11 on the test vehicle for damage detection. 


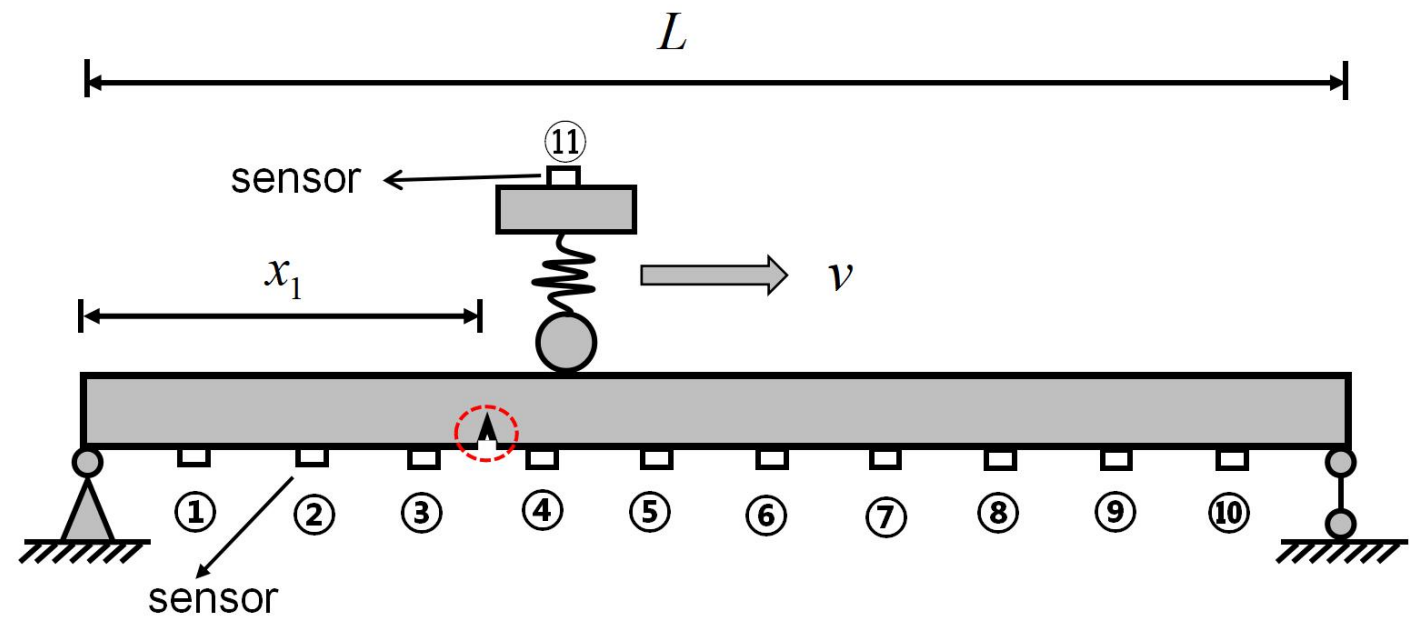

Figure 5. Schematic diagram of simply supported beam subjected to a test vehicle

The model parameters of simply supported beam bridges are as follows: the length, height, and width of beam are $L=50 m, h=0.2 m, b=0.1 m$, respectively. The elastic modulus and the unit mass length of beam are $E=30 G p a$ and $\bar{m}=7 \mathrm{~kg} / \mathrm{m}$, respectively.The physical parameters of the test vehicle are as follows: the mass and stiffness of the moving vehicle are $m_{v}=20 \mathrm{~kg}, k_{v}=251 \mathrm{kN} / \mathrm{m}$. And the road roughness is ignored.

The beam is equally divided into 50 beam units along the length of the beam. A measuring point is set on the measuring vehicle and a measuring point is set at any position of the 50 beam units of the beam. The vehicle is driven uniformly from the beginning of the beam to the end at velocities of $1,2,4$ and $6 \mathrm{~m} / \mathrm{s}$ respectively. The acceleration responses at measuring point 1-11 is collected and the sampling frequency is $800 \mathrm{~Hz}$. Using the moving window defined to scan these two responses step by step as shown in Figure 3. Damage units of bridge are characterized by stiffness reduction. The damage locations set at the positions of $L_{d}=0.3,0.4,0.6,0.7$, respectively. ( $L_{d}=x_{1} / L$ is the relative position between the damage position and the left end of the simply supported beam), is assumed in the simulations of single damage detection. The damage degree (loss rate of section) is 10\%, 30\% and 50\% respectively, as shown in Table 1.

Table 1. Scenarios of numerical simulation

\begin{tabular}{ccc}
\hline Damage location & Speed of the vehicle/(m/s) & Degree of damage \\
\hline $0.3 \mathrm{~L}$ & $1,2,4,6$ & $10 \%, 30 \%, 50 \%$ \\
$0.4 \mathrm{~L}$ & $1,2,4,6$ & $10 \%, 30 \%, 50 \%$ \\
$0.6 \mathrm{~L}$ & $1,2,4,6$ & $10 \%, 30 \%, 50 \%$ \\
$0.7 \mathrm{~L}$ & $1,2,4,6$ & $10 \%, 30 \%, 50 \%$ \\
\hline
\end{tabular}

Due to the structure's suddenly change, the acceleration responses signal also has singularity in the characterization information contained in the time before and after the test vehicle passes through the damage location. At the same time, the acceleration response of the numerical simulation at the measuring points of the test vehicle and bridge is collected to acquire the acceleration responses of the test vehicle and different measuring points under the action of moving load.

It can be seen from the previous section that damage location needs to calculate the transfer entropy and the length 
of moving window between the two sensors by acceleration data of vehicle-bridge system. For example, Figure 6(a) shows the acceleration response of the vehicle in undamaged states. The Fourier spectrum of the acceleration response of the undamaged beam is shown in Figure 6(b). As observed, the fundamental frequency of the response is $2.814 \mathrm{~Hz}$. The result of calculating window length is 569 according to equation (14), so that the window moves from left to right at the time of $t=0$ and the transfer entropy $M$ of acceleration data between two measuring points is calculated according to equation(9). Then the damage index $\mathrm{V}$ of the transfer entropy value is calculated by the distance equation (12) before and after damage, and the damage location is reflected by the peak value of the curves of damage index V.

Based on the concept of transfer entropy, the damaged measuring point data consists of two acceleration sensors, which obtain the vertical acceleration signal from one sensor at any point marked as 1 to 10 sensors on the bridge as shown in Figure 5 and the other one from the measuring point on the test vehicle marked as 11 sensor.

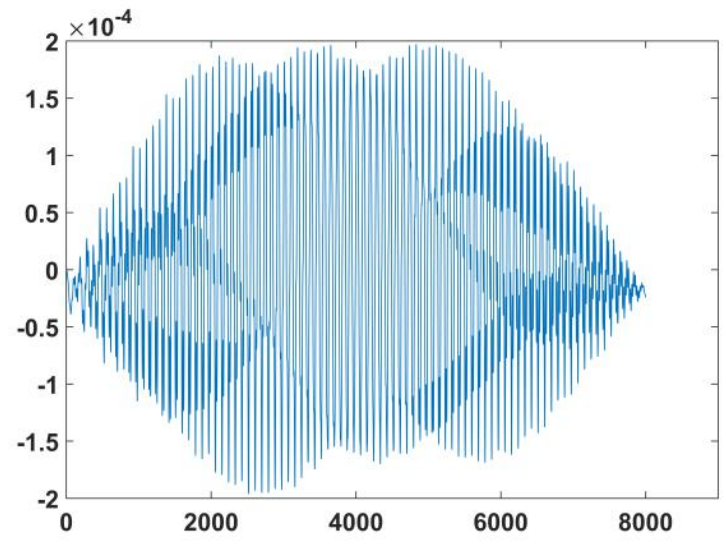

(a)

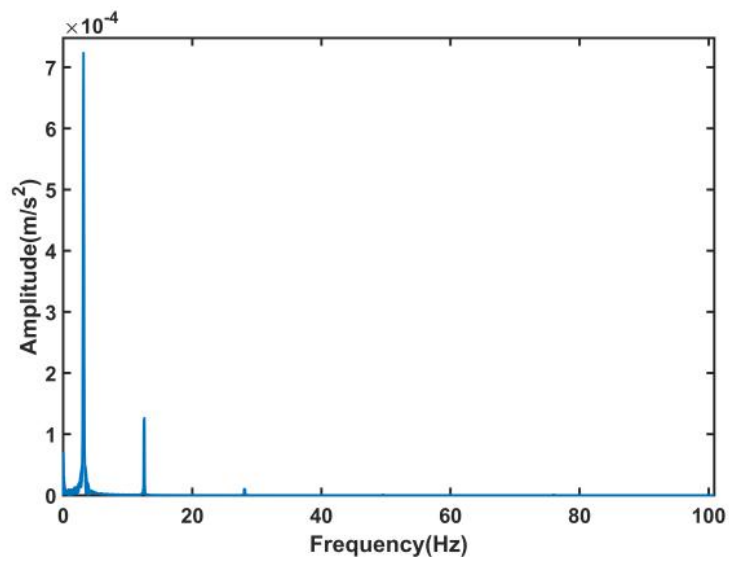

(b)

Figure 6. (a)Acceleration response of the test vehicle ; (b)Fourier spectrum of acceleration response of the undamaged beam

\subsection{Single damage detection}

In order to identify the accuracy of using the proposed damage index $\mathrm{V}$ to identify the damage using different sensor pairs, selecting the acceleration response of sensor1 and 7 on the bridge and sensors 11 on the test vehicle respectively. Using the moving window defined to scan these two responses obtained from the pair of sensors step by step, the damage detection result of $10 \%, 30 \%$ and 50\% damaged scenarios using sensors 1 and 11 is shown in Figure 7(a). And using sensors 7 and 11 is shown in Figure 7(b). Figure 7 shows the time series of the damage index V with damage degree of $10 \%, 30 \%$ and $50 \%$ respectively. As observed, all the curves of damage index using different pair of sensors have evident peaks at the damaged location at $L_{d}=0.6$. According to the concept of transfer entropy, it is speculated that the amount of information lost here is too large, indicating that there is damage here. All the curves of damage index $\mathrm{V}$ have a sudden peak at the damage location, and the damage index values increases with the increase of the damage degree. Therefore, this proposed approach can successfully locate the damage when selecting different sensor pairs. 


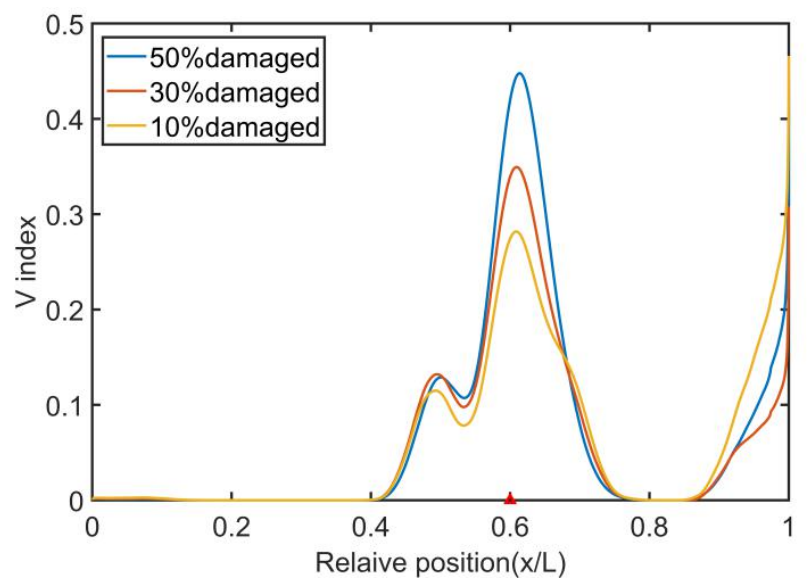

(a)

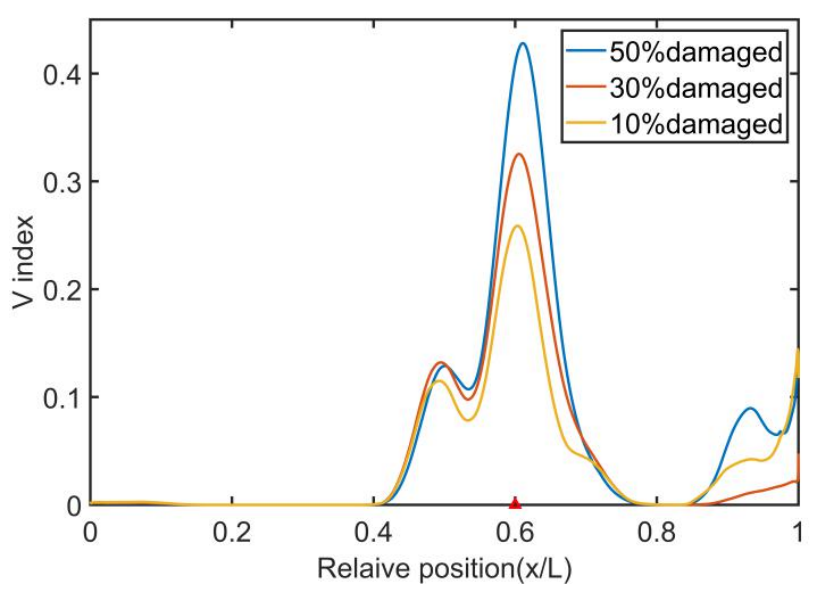

(b)

Figure 7. Damage detection results using different sensor pairs:(a)sensors 1 and 11;(b)sensors 7 and 11

In order to the verify the feasibility of using the proposed method to detect the damage at different locations, setting the damage at $L_{d}=0.4$ and $L_{d}=0.7$ respectively, the sensor 3 on the bridge and sensor 11 on the test vehicle are selected for damage identification. And the velocity of the test vehicle is $1 \mathrm{~m} / \mathrm{s}$. Figure 8 shows the curve of damage index $\mathrm{V}$ for $10 \%, 30 \%, 50 \%$ damaged scenarios using sensors 3 and 11 . The curves have peaks at position $L_{d}=0.4$ and $L_{d}=0.7$ respectively, which is the location of the damage set in the simulation. According to the different damage degrees and the peak value of the damage index at the damage location as show in the Figure 8 , as observed, the greater the bridge damage degree, and the greater the peak value.

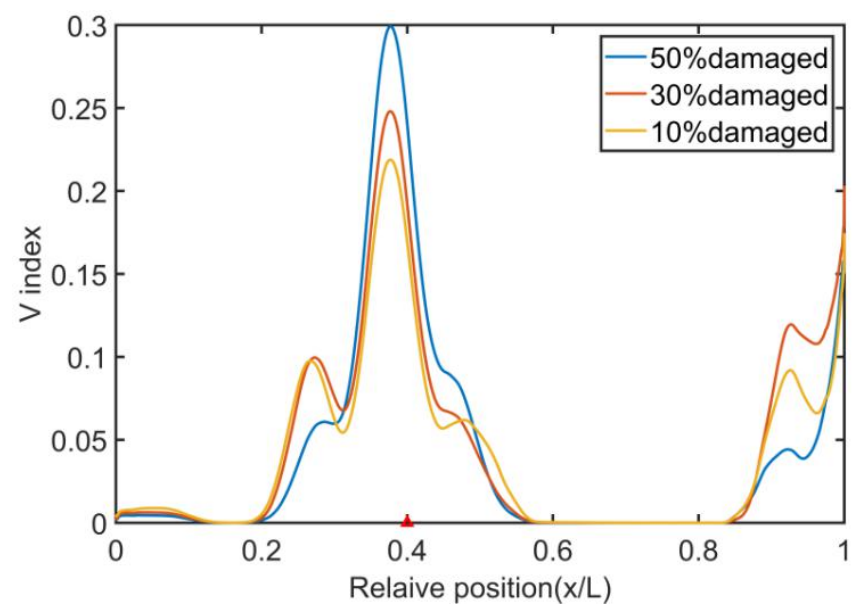

(a)

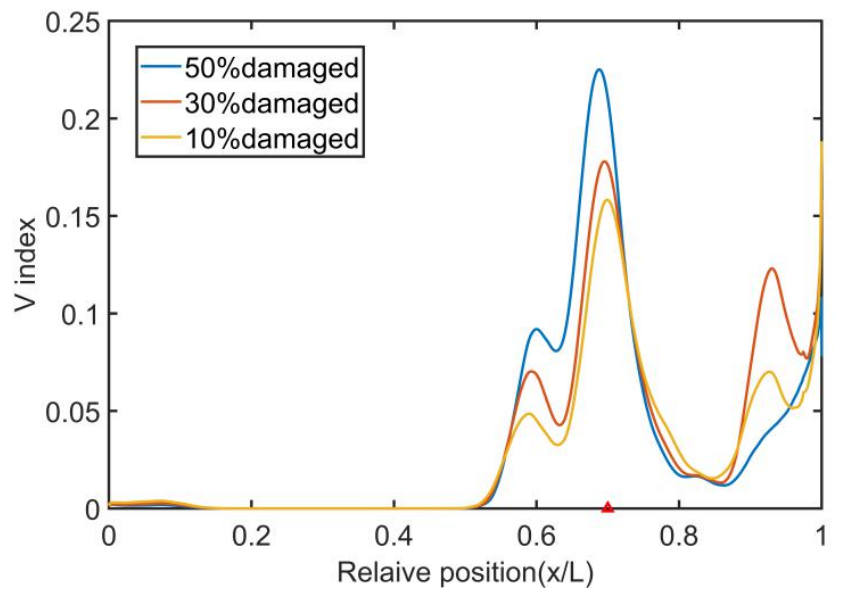

(b)

Figure 8. Damage detection results at a different damage location using sensors 3 and 11 : (a) ${ }^{L_{d}}=0.4$;(b) ${ }^{L_{d}=0.7}$

In order to study the influence of the velocity of the test vehicle on damage identification results, three different velocities of the test vehicle, namely 2,4 and $6 \mathrm{~m} / \mathrm{s}$, are defined. The damage location is set at $L_{d}=0.3$. Figure 9 shows the curve of damage index $\mathrm{V}$ for $10 \%, 30 \%, 50 \%$ damaged scenarios using sensors 4 and 11 . As observed, all the curves have a peak value at the location $L_{d}=0.3$, which is the location of the damage set in the simulation. 


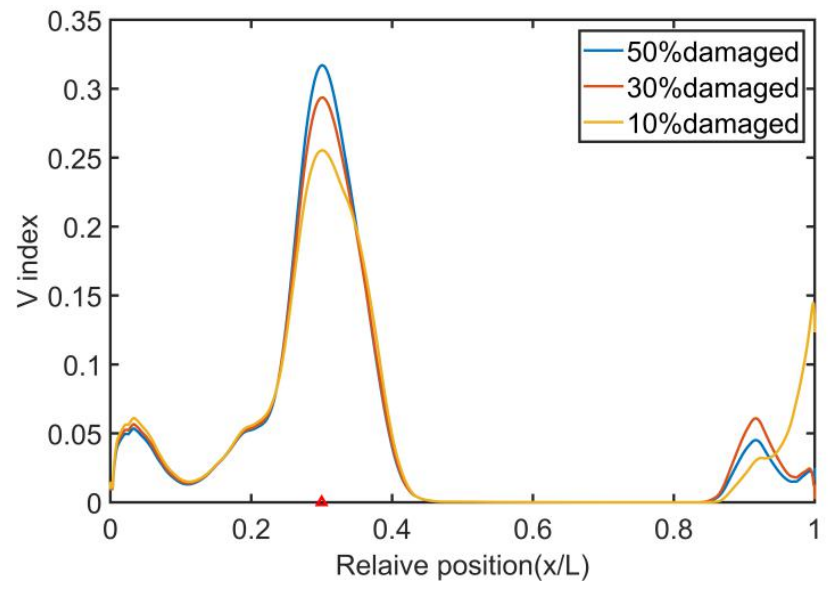

(a)

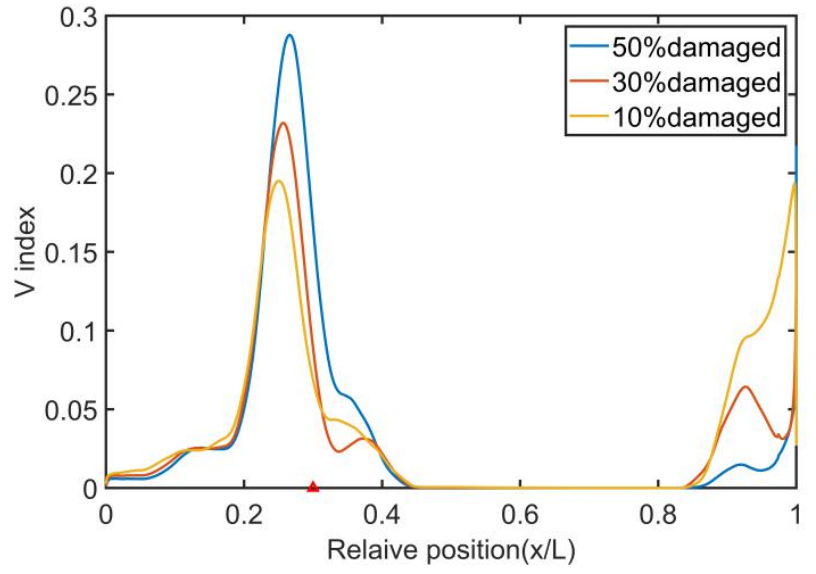

(b)

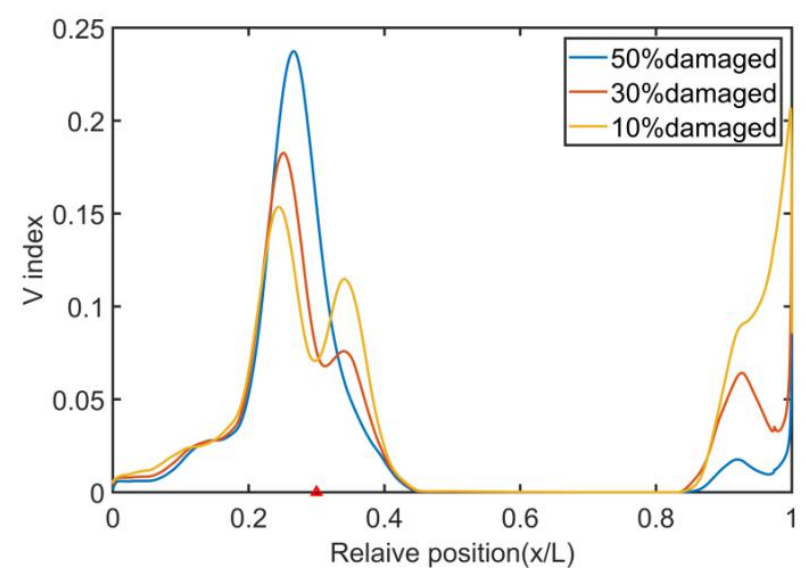

(c)

Figure 9. Damage detection results with different velocities of the test vehicle using sensors 4 and 11: (a) $2 \mathrm{~m} / \mathrm{s}$, (b) $4 \mathrm{~m} / \mathrm{s}$, and (c) $6 \mathrm{~m} / \mathrm{s}$.

By carefully comparing the three figures (a), (b) and (c) in Figure 9, it can be seen that when the velocity of vehicle is $2 \mathrm{~m} / \mathrm{s}$, the damage location can be accurately located even under damage degree of $10 \%$. When the velocity of vehicle is $4 \mathrm{~m} / \mathrm{s}$, the damage location can be located successfully under the damage degree of $30 \%$ and $50 \%$, while when the damage degree is $10 \%$, there is a deviation of about 0.02 between the peak of damage index curve and the damage position. When the velocity of the test vehicle increases to $6 \mathrm{~m} / \mathrm{s}$, the deviation occurs under the damage degree of $10 \%$ and $30 \%$. It shows that the sensitivity of this method is reduced when the vehicle velocity is large. This is because when the moving vehicle is loaded at a higher velocity, the vehicle crosses the bridge in a shorter time, so that the measured data length is inversely proportional to the velocity. The length of the moving window is determined by the sampling frequency and the fundamental frequency of the measured signal. Under the same conditions, the window length remains unchanged regardless of the velocity, which inevitably leads to a larger ratio of the data in the window to the whole measured signal and a lower resolution when the vehicle velocity is too high. The ability to capture local characteristic information of bridge decreases, which leads to the decrease of sensitivity of damage index when quantifying information transmission by window transfer Entropy method. Therefore, it is recommended that the moving velocity of vehicle should not be too high when using this method.

\subsection{Multiple damages detection}


In multiple damage scenarios, two damages at $L_{d}=0.3$ and $L_{d}=0.7$ are introduced in the model. In special cases, there may be several damaged locations of bridges. In order to demonstrate the validity of the proposed approach in various damaged scenarios, different degrees of damage are set at damaged locations of $0.3 \mathrm{~L}$ and $0.7 \mathrm{~L}$ at the same time. The velocity of the test vehicle is $1 \mathrm{~m} / \mathrm{s}$, and the acceleration responses measured by different sensor pairs. As show in Figure 10 (a), is the damage detection results using sensors 4 and 11, and Figure 10(b) is the damage detection results using sensors 9 and 11. The results show that the method can well detect the damage in multiple damage scenarios. All the curves of damage index $\mathrm{V}$ for different damage severities have evident peaks at the damaged location, which is the location of the damage set in the simulation. Based on the concept of transfer entropy, the information loss at these two locations is the largest. With the increasing damage severity, the value of $\mathrm{V}$ also increases with the damage severity.

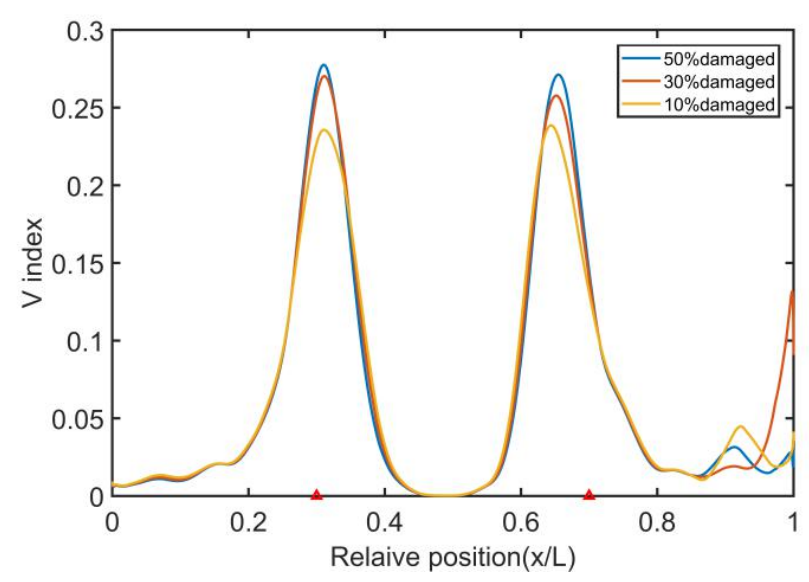

(a)

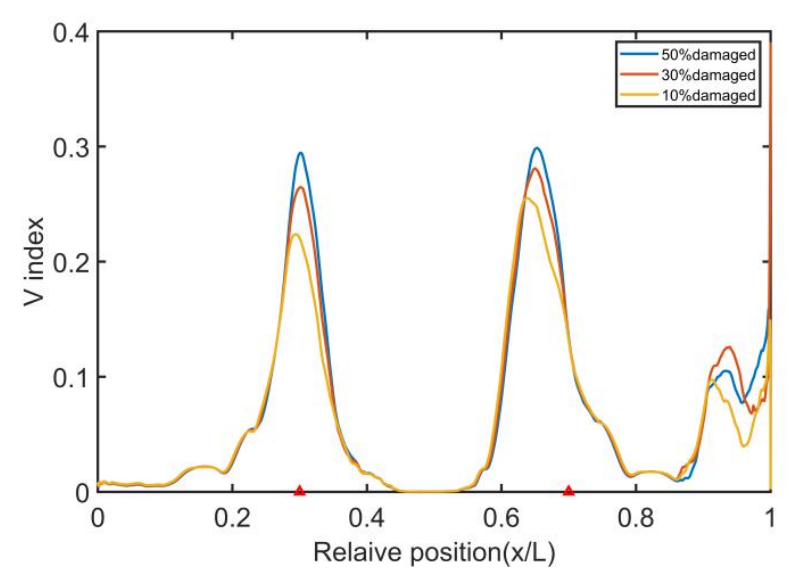

(b)

Figure 10. Damage detection results of multiple damage scenarios using different sensor pairs:(a)sensor 4 and 11, sensor 9 and 11.

\section{Conclusions}

In this article, an innovative damage identification approach based on indirect measurement method and transfer entropy theory using only two sensors of the vehicle-bridge information is proposed. By installing one sensor on the vehicle and one sensor at any location on the bridge, i.e., the method of installing two sensors for damage identification of the bridge. According to the transfer entropy theory, there is a difference in the amount of information between the data of two measurement points located on the bridge and on the test vehicle before and after the structural damage, and the damage is localized by calculating the magnitude of the missing information before and after the damage. A fixed moving window is defined as a definitive length calculated by the sampling frequency and the fundamental frequency of the measured acceleration responses. The windowed pair time series extracted from the two measured acceleration responses that obtained from the bridge and the moving vehicle respectively are used to calculate the transfer entropy, which is used to define the new damage index. The damage is localized by the peak of the damage index. The numerical simulation results indicate that this proposed approach is able to accurately locate the damage under both single damage scenarios and multiple damages scenarios, and the peak value of damage index at the damage location increases with the increase of damage degree. However, when the vehicle speed is large, the sensitivity of this method decreases. Therefore, the velocity of the test vehicle should not be too high, much more work should carry out to further verify the effectiveness of the proposed approach. 


\section{Conflicts of Interest}

The author declares no conflicts of interest regarding the publication of this article.

\section{Acknowledgments}

The authors acknowledge the financial supports from the projects in key areas of Guangdong Province (No.2019B111106001) and National Key Research and Development Project of China (No. 2019YFC1511000).

\section{References}

[1] Zhu X.Q., \& Law S.S. (2006). Wavelet-based Crack Identification of Bridge Beam from Operational Deflection Time History. International Journal of Solids and Structures, 43(7-8):2299-2317.

[2] Nie Z., Ngo T., \& Ma H. (2017). Reconstructed Phase Space-based Damage Detection Using a Single Sensor for Beam-like Structure Subjected to a Moving Mass. Shock and Vibration,(2):1-20.

[3] Li J., \& Law S. S. (2012). Damage Identification of a Target Substructure with Moving Load Excitation. Mechanical Systems \& Signal Processing, 30, 78-90.

[4] Li J., Law S.S., \& Hao H. (2013). Improved Damage Identification in Bridge Structures Subject to Moving Loads: Numerical and Experimental Studies. International Journal of Mechanical Sciences, 74, 99-111.

[5] Yang Y.B., Lin C.W., \& Yau J.D. (2004). Extracting Bridge Frequencies from the Dynamic Response of a Passing Vehicle. Journal of Sound and Vibration, 272(3-5), 471-493.

[6] Yang Y.B., Lin C., \& W. (2007). Vehicle-bridge Interaction Dynamics and Potential Applications. Noise \& Vibration Worldwide, 38(2), 30-31.

[7] Bu J.Q., Law S S, \& Zhu X.Q. (2006). Innovative Bridge Condition Assessment from Dynamic Response of a Passing Vehicle[J]. Journal of Engineering Mechanics, 132(12),1372-1379.

[8] Nguyen, K.V., \& Hai T.T. (2010). Multi-cracks Detection of a Beam-like Structure Based on the on-vehicle Vibration Signal and Wavelet Analysis. Journal of Sound \& Vibration, 329(21), 4455-4465.

[9] Aoyagi, S., Kawashima, Y., \& Kudo, M. (2005). Tof-sims Imaging Technique with Information Entropy. Nuclear Instruments \& Methods in Physics Research, 232(1-4), 146-152.

[10] Schreiber, \& Thomas. (2000). Measuring Information Transfer. Physical Review Letters, 85(2), 461-464.

[11] Nichols, J. M., Seaver, M., \& Trickey, S. T. (2006). A Method for Detecting Damage-induced Nonlinearities in Structures Using Information Theory. Journal of Sound \& Vibration, 297(1-2), 1-16.

[12] Dimpfl, T., \& Peter, F.J. (2018). Analyzing Volatility Transmission Using Group Transfer Entropy. Energy Economics, 75.

[13] Yang Y.B. , \& Chang K.C. (2009). Extraction of Bridge Frequencies from the Dynamic Response of a Passing Vehicle Enhanced by the end Technique. Journal of Sound and Vibration, 322(4-5), 718-739.

[14] Yang Y., B., Lin, C., \& W. (2007). Vehicle-bridge Interaction Dynamics and Potential Applications. Noise \& Vibration Worldwide, 38(2), 30-31.

[15] Nichols, J. M., Seaver, M., Trickey, S.T., Salvino, L.W., \& Pecora, D.L. (2006). Detecting Impact Damage in Experimental Composite Structures: an Information-theoretic Approach. Smart Materials \& Structures, 15(2), 424.

[16] Overbey, L.A., \& Todd, M. (2009). Dynamic System Change Detection Using a Modification of the Transfer Entropy. Journal of Sound and Vibration, 322(1-2), 438-453.

[17] Laura, P., Rossi, R. E., Yang Y.B., \& Lin B.H. (1997). Discussion and Closure: Vehicle-bridge Interaction Analysis by Dynamic Condensation Method. Journal of Structural Engineering, 123(4), 532-533. 